\title{
Different Gating Mechanisms in Glutamate Receptor and $\mathrm{K}^{+}$Channels
}

\author{
Alexander I. Sobolevsky, Maria V. Yelshansky, and Lonnie P. Wollmuth \\ Department of Neurobiology and Behavior, State University of New York at Stony Brook, Stony Brook, New York 11794-5230
}

The basic structural features of channel gating in glutamate receptors (GluRs) remain unknown. Here we used covalent modification of substituted cysteines and fast agonist application to study the contribution of the M3 segment in AMPA receptor GluR-A subunits to channel structure and gating. The pattern of accessibility of substituted cysteines to extracellularly applied methanethiosulfonate reagents and the rates of their modification by these reagents, measured in either the presence or absence of glutamate, indicate that M3 forms an $\alpha$-helix that lines the pore of the channel and is involved in gating-related movements. The voltage dependence of modification rates places the tip of the M2 loop (the Q/R site) close to the middle of M3. All of these results are consistent with pore-forming domains in GluR and $\mathrm{K}^{+}$channels having a similar structure but inverted membrane topology. Nevertheless, GluRs lack a glycine residue at a homologous structural position as the gating hinge glycine in $\mathrm{K}^{+}$channels. Moreover, simultaneous substitution of the only two glycines in M3 of GluR-A with alanines produced channels with gating properties indistinguishable from wild type. Given the unique role of glycines in the flexibility of $\alpha$-helices, our results indicate that the M3 segment in GluR does not contain a glycine gating hinge and suggest that, in contrast to the homologous domain in $\mathrm{K}^{+}$channels, $\mathrm{M} 3$ is rigid during gating. The different positioning and functional significance of glycines in a key structural domain may represent the basis for the distinct features of gating in $\mathrm{GluR}_{\text {and }} \mathrm{K}^{+}$channels.

Key words: glutamate receptors; SCAM; reaction rates; pore-forming domains; $\mathrm{K}^{+}$channels; gating hinge; voltage dependence; MTS reagents; accessibility

\section{Introduction}

A key function of ionotropic glutamate receptors (GluRs) in synaptic physiology is defined by channel gating: the conversion of the conformation changes in the ligand-binding domain after glutamate binding to channel opening. Recent crystallographic studies have revealed essential features of gating at the level of the ligand-binding domain (Armstrong et al., 1998; Armstrong and Gouaux, 2000; Mayer et al., 2001; Sun et al., 2002). In contrast, the structural basis of how conformational changes in the ligandbinding domain are propagated to transmembrane segments and correspondingly to gating of the ion conduction pathway remains poorly understood.

GluR channels share general structural features with $\mathrm{K}^{+}$ channels (see Fig. 1A) (for review, see Kuner et al., 2003). In $\mathrm{K}^{+}$ channels, the inner helix (M2) is the major structural element lining the intracellular vestibule and defining gating. In the closed state, M2 is nearly straight and forms a gate for $\mathrm{K}^{+}$ions at the intracellular mouth of the channel (Liu et al., 1997; del Camino and Yellen, 2001). With channel opening, M2 bends away from the central axis of the pore at the point of a gating hinge formed by a highly conserved glycine (Jiang et al., 2002b). This glycine is

\footnotetext{
Received April 30, 2003; revised June 18, 2003; accepted July 3, 2003.

This work was supported by National Institutes of Health Grant R01 NS39102 and the American Parkinson's Disease Association (L.P.W.). We thank Drs. Mark L. Mayer, Gary Matthews, and Markus Eilers for comments on this manuscript. We thank Dr. Paul Brehm for help with Xenopus laevis.

Correspondence should be addressed to Dr. Alexander I. Sobolevsky, Department of Neurobiology and Behavior, State University of New York at Stony Brook, Stony Brook, NY 11794-5230. E-mail: asobolevsky@notes2.cc.sunysb.edu. Copyright $\odot 2003$ Society for Neuroscience $\quad 0270-6474 / 03 / 237559-10 \$ 15.00 / 0$
}

positioned just below the tip of the P loop, permitting movement of M2 independent of the P loop and leaving the selectivity filter essentially motionless during gating. The positioning of this gating hinge glycine is critical because even a slight change in it disrupts gating, in most cases yielding nonfunctional channels (Ding and Horn, 2003).

The homologous domain in GluRs, the M3 segment, also contributes to channel gating (Kohda et al., 2000; Jones et al., 2002; Sobolevsky et al., 2002a). Surprisingly, in a sequence alignment (see Fig. $1 B$ ), GluR M3s have no glycine at the position homologous to the gating hinge in $\mathrm{K}^{+}$channels, although two glycines are located 6 and 7 positions $\mathrm{N}$-terminal. To address the general topology of pore-forming domains in GluRs and a potential role of glycines in gating, we took advantage of covalent modification of substituted cysteines as well as fast agonist application. In these experiments, we focused on the M3 segment in AMPA receptors (AMPARs) because they can form homomultimers, like $\mathrm{K}^{+}$ channels, and represent the most likely GluR subtype to show structural and functional homology to them.

We find that the M3 segment in AMPARs composed of GluR-A (GluR1) subunits is a pore-lining $\alpha$-helix involved in channel gating. The tip of the M2 loop is positioned close to the middle of M3. Although similar to $\mathrm{K}^{+}$channels, this structural arrangement places no glycine residue in $\mathrm{M} 3$ right above or even close to the tip of the M2 loop. Furthermore, substitution of two glycines in M3 not only produced functional channels [in contrast to $\mathrm{K}^{+}$channels (Yifrach and MacKinnon, 2002)], but the channels displayed gating properties indistinguishable from wild 
type. We therefore propose that M3 in GluRs does not contain a glycine gating hinge and that, in contrast to $\mathrm{K}^{+}$channels, gating in GluRs involves a rigid M3.

\section{Materials and Methods}

\section{Mutagenesis and heterologous expression}

All cysteine substitutions were introduced into a GluR-A (flip form) expression construct in which a leucine in the ligand-binding domain was substituted with a tyrosine [GluR-A(L479Y)]. This construct is essentially nondesensitizing (Stern-Bach et al., 1998). Point mutations were generated either with a QuickChange site-directed mutagenesis kit (Stratagene, La Jolla, CA) or by other PCR-based methods using Platinum Pfx DNA polymerase (Invitrogen, Carlsbad, CA) or Pfu poly DNA polymerase (Stratagene, La Jolla, CA). Positive clones were subcloned back into the respective wild-type clone, either in the pSP vector or the eukaryotic expression vector pRK. All constructs were sequenced over the entire length of the replaced fragment. cRNA was transcribed and capped for each expression construct using SP6 RNA polymerase (Ambion, Austin, TX) and examined electrophoretically on a denaturating agarose gel. RNA concentrations were determined by ethidium bromide stain of the gel relative to an RNA molecular weight marker. Dilutions of RNA $(0.01-0.1 \mu \mathrm{g} / \mu \mathrm{l})$ were prepared to achieve optimal expression. Nondesensitizing wild-type [GluR-A(L479Y) or wt'] or cysteinesubstituted subunits were expressed in Xenopus laevis oocytes. Oocytes were prepared, injected, and maintained as described (Wollmuth et al., 1996; Sobolevsky et al., 2002a). Recordings were made 1-6 d after injections.

Wild-type and mutant GluR-A subunits (without the nondesensitizing L479Y mutation) were also expressed transiently in human embryonic kidney 293 (HEK 293) cells. Cells were transfected using the "adherent cells" procedure as outlined by the manufacturer (FuGene 6, Roche, Indianapolis, IN) at a ratio of $100 \mu \mathrm{m}$ FuGene/ $1 \mu \mathrm{g}$ transfected DNA. A vector for enhanced green fluorescent protein (pEGFP-C1; Clontech, Palo Alto, CA) was cotransfected at a ratio of 1:9 (GFP/GluR-A). Recordings were made $1-4 \mathrm{~d}$ after transfection.

\section{Current recordings and data analysis}

Whole-cell currents of Xenopus oocytes were recorded at room temperature $\left(20-23^{\circ} \mathrm{C}\right)$ using two-electrode voltage-clamp (DAGAN TEV200A, DAGAN Corporation, Minneapolis, MN) with Cell Works software (npi electronic, Tamm, Germany). Microelectrodes were filled with $3 \mathrm{M} \mathrm{KCl}$ and had resistances of 1-4 M $\Omega$. To minimize solution exchange rates, we used a narrow flow-through recording chamber with a small volume of $\sim 70 \mu \mathrm{l}$. The external solution consisted of $(\mathrm{mm}): 115 \mathrm{NaCl}$, $2.5 \mathrm{KCl}, 0.18 \mathrm{CaCl}_{2}$, and $10 \mathrm{HEPES}, \mathrm{pH} 7.2, \mathrm{NaOH}$. Glutamate (1 mM), $\mathrm{CNQX}$, and methanethiosulfonate (MTS) reagents were applied with the bath solution.

Currents in HEK 293 cells were recorded at room temperature (20$23^{\circ} \mathrm{C}$ ) using an EPC-9 amplifier with PULSE software (HEKA Elektronik, Lambrecht, Germany), low-pass filtered at $2.9 \mathrm{kHz}$, and digitized at 10 $\mathrm{kHz}$. Patch pipettes had resistance of $2-4 \mathrm{M} \Omega$ when filled with the pipette solution and measured in the extracellular solution. No series resistance compensation was used. External solutions were applied using a piezodriven double-barrel application system. One barrel contained the external solution, whereas the other barrel contained the same solution with added glutamate ( $1 \mathrm{~mm}$ ). The $10-90 \%$ rise time of the open tip response measured with this application system was $\sim 350 \mu \mathrm{sec}$. This time was close to the rise time of glutamate-activated currents in outside-out patches $(300-500 \mu \mathrm{sec})$, suggesting that to a great extent the activation process was limited by the rate of solution exchange. The intracellular (pipette) solution consisted of (in $\mathrm{mM}$ ): $140 \mathrm{KCl}, 10 \mathrm{HEPES}$, and 1 BAPTA, pH 7.2, KOH. The extracellular solution consisted of (mM): 140 $\mathrm{NaCl}, 10 \mathrm{HEPES}, 1.8 \mathrm{CaCl}_{2}$, and $1 \mathrm{MgCl}_{2}, \mathrm{pH} 7.2, \mathrm{NaOH}$.

Data analysis was done using Igor Pro (WaveMetrics, Lake Oswego, OR) and Microcal Origin 4.1 (Northampton, MA). For analysis and display, leak currents were subtracted from total currents. Results are presented as mean \pm SEM. An ANOVA or a Student's $t$ test was used to test for statistical differences. The Tukey test was used for multiple comparisons. Significance was assumed if $p<0.05$.

\section{Experimental protocols}

AMPAR cysteine-substituted mutant channels were probed from the extracellular side of the membrane with MTS reagents: 2-aminoethyl MTS (MTSEA) and 2-(trimethylammonium)ethyl MTS (MTSET). MTS-containing solutions were prepared, stored, and applied as described (Sobolevsky et al., 2002a). MTS reagents were purchased from Toronto Research Chemicals (Ontario, Canada). All other chemicals were obtained from Sigma (St. Louis, MO).

Steady-state reactions. Steady-state reactions were quantified at $-60 \mathrm{mV}$ (see Fig. 2). Baseline glutamate-activated current amplitudes $\left(I_{\text {pre }}\right)$ were established by three to five consecutive $15 \mathrm{sec}$ applications of glutamate separated by $60-120 \mathrm{sec}$ washes in glutamate-free solution. Subsequent to the last wash, an MTS reagent ( $2 \mathrm{mM})$ was applied for $60 \mathrm{sec}$ either in presence of glutamate or in its absence (but in the presence of $10 \mu \mathrm{M}$ CNQX). After the MTS exposure, current amplitudes $\left(I_{\text {post }}\right)$ were determined again using three to five glutamate applications. The washout interval between the end of the MTS reagent application and the first post-glutamate application ranged from 1.25 to $5 \mathrm{~min}$. The change in the glutamate-activated current amplitude, expressed as a percentage $(\%$ change $)$, was calculated as $=\left(1-I_{\text {post }} / I_{\text {pre }}\right) \times 100$. The change in the leak current amplitude, expressed as a percentage $(\Delta$ leak $)$, was calculated as $=$ $\left(\left(I_{\text {post }}^{\text {leak }}-I_{\text {pre }}^{\text {leak }}\right) /\left(I_{\text {pre }}+I_{\text {pre }}^{\text {leak }}\right)\right) \times 100$, where $I_{\text {pre }}^{\text {leak }}$ and $I_{\text {post }}^{\text {leak }}$ are the leak current amplitudes before and after the MTS reagent application, respectively.

Reaction rates. Reaction rates $(k)$ were determined by using a "pulsive" protocol (see Fig. 3) as described in detail in Sobolevsky et al. (2002b). The voltage dependence of $k$ was analyzed according to the following equation:

$$
k=k_{0} \exp \left(-z \delta F V_{\mathrm{h}} / R T\right)
$$

where $V_{\mathrm{h}}$ is the holding potential, $k_{0}$ is the apparent second-order rate constant for modification at $V_{\mathrm{h}}=0, \delta$ is the fraction of the transmembrane electric field that the MTS reagent passes to reach the exposed cysteine, and $z$ is the charge of the reagent. $F, R$, and $T$ and Faraday's constant, the gas constant, and the absolute temperature, respectively. To derive $z \delta$, we rearranged Equation 1 :

$$
-(R T / F) \operatorname{Ln} k=A+z \delta V_{\mathrm{h}}
$$

where $A$ is $-(R T / F) \operatorname{Ln} k_{0}$, and fitted Equation 2 to plots of $-(R T / F) \operatorname{Ln} k$ against $V_{\mathrm{h}}$.

Gating kinetics. To determine gating kinetics, we recorded currents at $-60 \mathrm{mV}$ in outside-out patches excised from HEK 293 cells. Desensitization $\left(\tau_{\text {des }}\right)$ and deactivation $\left(\tau_{\text {deact }}\right)$ time constants were determined by fitting the current decay during $100 \mathrm{msec}$ and after $1 \mathrm{msec}$ application of glutamate, respectively, with a single exponential function. The steadystate to peak current ratio (SS/peak ratio) was calculated as $I_{\mathrm{SS}} / I_{\mathrm{CTZ}}$, where $I_{\mathrm{SS}}$ is the steady-state current amplitude in the presence of glutamate $(1 \mathrm{mM})$ and $I_{\mathrm{CTZ}}$ is the maximal current amplitude in the presence of glutamate and $30 \mu \mathrm{m}$ cyclothiazide. The time constant for recovery from desensitization $\left(\tau_{\text {rec }}\right)$ was estimated using a two-pulse protocol. After a base $100 \mathrm{msec}$ application of glutamate, a $5 \mathrm{msec}$ test application of glutamate was applied at increasing times thereafter. The peak during the test application $\left(I_{\text {test }}\right)$ was normalized to the peak current of the base application $\left(I_{\text {base }}\right): I_{\text {norm }}=I_{\text {test }} / I_{\text {base }}$. Normalized currents were plotted as a function of time after the end of the base application and fitted with a single exponential function to derive $\tau_{\text {rec }}$.

\section{Modeling}

To illustrate our general ideas, we built simplified three-dimensional geometrical models of GluR and $\mathrm{K}^{+}$channels using the POV-Ray (version 3.5 for Windows) program. In these models, $\alpha$-helical regions are represented by cylinders (diameter $5 \AA$; the ends were defined on the basis of the coordinates of the beginnings and ends of the corresponding $\alpha$-helical regions) connected by loops (cubic splines on the basis of coordinates of $\mathrm{C}_{\alpha} \mathrm{s}$ in the extended regions). $\mathrm{K}^{+}$channel models (see Fig. 7) were made on the basis of coordinates of KcsA [Protein Data Bank (PDB) code 1K4C] and MthK (PDB code 1LQN) channels (Zhou et al., 2001; Jiang et al., 2002a). GluR channel models were generated by transform- 
A

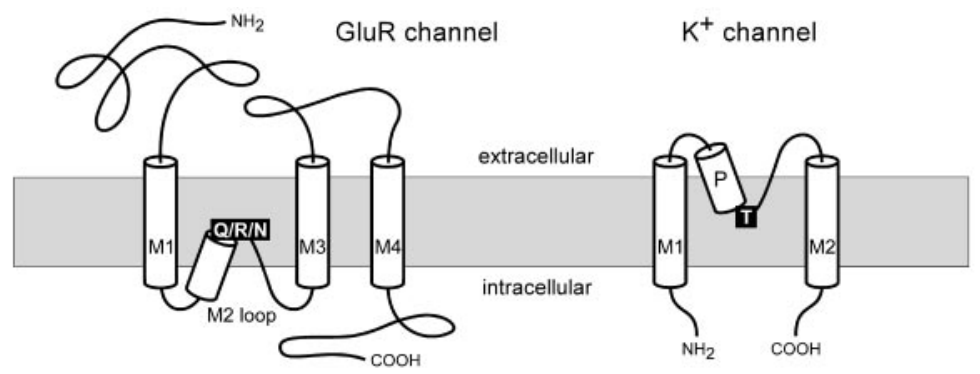

B

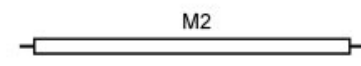

GluR-A 564 SNEFGIFNSLWESLGAFN

GluR-6 572 ENNFTLLNSEWEGVGALM

KA1 568 VNQYSLGNSLWEPVGGEM

NR1 580 EDALTLSSAMWESWGVLI

NR2A 577 GPSETIGKAIWLLWGLVE

NR2C 575 GPSFTIGKSVWLLWALVP

NR3A 575 NKVESFSSALNVCYALLE

GluR0

5 NKVESFSSALNVCYALLE

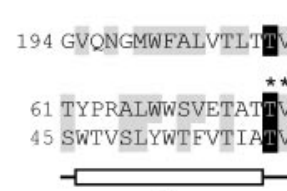

$\mathrm{P}$

$\mathrm{KcsA}$

MthK

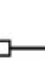

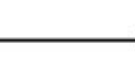

M3

CCCCCCCCCCCCCCCCCCCCCCCCCCCCCCCC

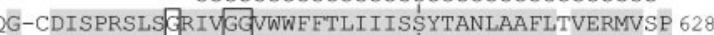
OG-SELMPKALSTRIVGGIWWFFTLI I ISSYTANLAAFLTVERMESP 637 QQG-STIAPRALSTRCVSGVWWAFTLI I ISSYYTANLAAFLTVQRMEVP 632 SGIGEGAPRSESARIIGMVWA GFMI IVASYTANLAAFLVLDRPEER 645 NSVPVQNPKG ITSKIMVSVWAFFAVI FLASYTANLAAFMIQEEFVDQ 642 NSVPIENPRGTTSKIMVLVWAFFAVI FLASYTANLAAFMIQEQYIDT 640 RTAAIKPPKCWTGRFLMNWAI ECMFCLSTYTANLAAVMVGEKIYEE 640 $\begin{array}{llllll}-15 & -10 & -5 & 0 & -5 & +\end{array}$

VGYGDRSPRTKIGQLVAGVWMLVALLSESSITAGLASAFST 249

$\star \star \star \star \star \star$ $\checkmark \sqrt{2}$

VGYGDLYPVTLWGRLVAVVVMVAGITSFGLVTAALATWFVG 116 GYGDYSPSTPLGMYETVTLIVLGIGTFAVAVERLLEFLIN 100

M2

Figure 1. Comparison of GluR and $\mathrm{K}^{+}$channel subunits. $A$, Membrane topology. Presumed membrane-spanning $\alpha$-helical domains in GluR subunits (M1, M3, and M4) and definitive comparable domains in $\mathrm{K}^{+}$channel subunits (M1 and M2) are shown as white cylinders. Black boxes indicate the tip of the reentrant loop, Q/R/N site in GluR channels, and T site in $\mathrm{K}^{+}$channels. Domains containing M1 to M3 in GluR subunits have a similar but inverted membrane topology to $\mathrm{K}^{+}$channel subunits. $B$, Sequence alignment of pore-forming domains. Shown are amino acid sequences for AMPAR (GluR-A), KAR (GluR-6 and KA1), NMDAR (NR1, NR2A, NR2C, and NR3A), prokaryotic GluR0, and $\mathrm{K}^{+}$channel (KcsA and MthK) subunits. Open boxes in the schematic drawings above and below the sequence alignment indicate hydrophobic segments $M 2$ and $M 3$ in GluR subunits and the pore helix (P) and inner helix (M2) in $\mathrm{K}^{+}$channel subunits, respectively. Positions in GluR-A substituted with cysteines and alanines in the present study are indicated (CC. . . CC and AA, respectively). For ease of comparison, amino acids are numbered relative to the first position $(S)$ in SYTANLAAF, the most highly conserved motif in GluR. The residues located at the tip of the reentrant loops [the N site in NMDARs (Kuner et al., 1996) and Q/R site in AMPARs (Kuner et al., 2001)] are shown in black boxes. In $\mathrm{K}^{+}$channel subunits, the amino acids that form the selectivity filter are indicated by asterisks, whereas those that correspond to the narrowest point of the MthK intracellular entryway and the gating hinge glycines are indicated by white and black arrows, respectively (Jiang et al., 2002a). Open boxes indicate glycines in GluR subunits located in M3 and in the M2-M3 linker. Other positions occupied by similar residues are highlighted in gray.

ing the $\mathrm{K}^{+}$channel models to account for the following conditions: (1) the diameter of the GluR channel narrow constriction in the closed state is $<3 \AA$ to prevent ion flux; (2) the diameter of the narrow constriction in the open state is $\sim 7 \AA$ (Burnashev et al., 1996); (3) the symmetry and general arrangement of the transmembrane domains and the general direction of their movements during gating is similar to those in $\mathrm{K}^{+}$ channels; (4) the movement of M2 loops during gating parallels the movement of the transmembrane domains; and (5) there is no gating hinge in the M3 segment.

\section{Results}

We used the substituted cysteine accessibility method (SCAM) to determine the general structure of the M3 segment and its positioning relative to $\mathrm{M} 2$. The interpretation of our results is limited by the assumptions of SCAM (Karlin and Akabas, 1998). For example, we assume that the cysteine substitution itself does not alter significantly the structure of the protein. We also consider a substituted cysteine to be exposed to the water interface and presumably to the lumen of the channel if glutamate-activated currents are persistently altered after the application of an MTS reagent. We also assume that a lack of a persistent effect on glutamate-activated currents indicates that the substituted cysteine is not in a water interface, presumably because it is buried in the interior of the protein. In certain instances, exposed cysteines may not be modified or may be modified without producing a persistent effect (silent reaction).

For our experiments, we generally used as a control and background the nondesensitizing GluR-A(L479Y) (wt') AMPAR subunit (Stern-Bach et al., 1998). We assume that channels composed of these subunits exist primarily in the closed state (absence of glutamate) or in the closed and open states (presence of glutamate). Residues within and C-terminal to the M3 segment as well as the $Q / R$ site glutamine (Q582) in the M2 loop were individually mutated to cysteine (Fig. $1 B$ ). To simplify comparison with other GluR subunits, we referenced positions in M3 relative to the initial residue (S) of the highly conserved SYTANLAAF motif. When expressed in Xenopus oocytes, 27 of 33 cysteinesubstituted AMPAR mutants generated glutamate-activated currents comparable in amplitude with wt', 5 (W-10C, W-9C, $\mathrm{F}-7 \mathrm{C}, \mathrm{L}+5 \mathrm{C}$, and $\mathrm{Q} 582 \mathrm{C})$ generated smaller currents, and $1(\mathrm{Y}+1 \mathrm{C})$ did not generate detectable current. Leak currents for cysteine-substituted mutant channels before application of MTS reagents were comparable in amplitude with that for wt' with one exception, $\mathrm{A}+3 \mathrm{C}$, which showed much larger leak current.

\section{Accessibility of substituted cysteines to MTS reagents}

Figure $2 A$ illustrates our protocol to determine accessibility of substituted cysteines in the presence of glutamate. In the example shown, GluR-A(L479Y) (wt') channels, glutamate-activated currents recorded before $\left(I_{\text {pre }}\right)$ and after $\left(I_{\text {post }}\right)$ exposure to MTSET ( $2 \mathrm{~mm}$, thick line) applied in the continuous presence of glutamate ( $1 \mathrm{~mm}$, thin line) were unchanged, indicating that any possible modification of endogenous cysteines does not affect glutamate-activated currents. Similar results were obtained for wild-type GluR-A channels not containing the L479Y mutation (e.g., for MTSET applied in the presence of glutamate and $50 \mu \mathrm{M}$ cyclothiazide, $\%$ change $=1.3 \pm 2.5 \% ; n=3$; mean \pm SEM). To determine accessibility of substituted cysteines in the absence of glutamate, we used a comparable protocol (Fig. 2 B), except that we applied MTSEA in the absence of glutamate and in the continuous presence of the competitive AMPAR antagonist CNQX (10 $\mu \mathrm{M}$, open box) to minimize the probability of channel openings.

Figure $2 C$ summarizes the mean percentage change in current amplitudes measured before and after exposure to MTSEA (MTSEA + Glu) or MTSET (MTSET + Glu) in the presence of glutamate or MTSEA in the absence of glutamate (MTSEA - Glu). Filled bars indicate positions where $I_{\text {post }}$ was significantly different from $I_{\text {pre }}$. On the basis of the assumptions of SCAM, we considered such positions accessible to reaction with MTS reagents and as a first approximation exposed to the water interface and lining the channel pore. For accessible positions, MTS re- 
agents typically reduced glutamateactivated current amplitudes. In two instances, however, I-2C and S-1C, MTSEA had biphasic effects on glutamateactivated current amplitudes, a fast potentiation and a slow inhibition. For S-1C, the inhibition occurred so slowly that during a single MTSEA application (Fig. 2A) currents were only potentiated (Fig. 2C, MTSEA + Glu) (during a second MTS application, current inhibition started). On the other hand, for $\mathrm{I}-2 \mathrm{C}$ the fast potentiation was over during a single MTS application, yielding a net current inhibition (Fig. $2 C$, MTSEA + Glu).

Figure 2C (MTSEA + Glu) shows that the entire M3 segment contains positions accessible to MTSEA in the presence of glutamate. This pattern is different from those for NMDAR subunits, where positions only in the C-terminal (extracellular) half of M3 are accessible to MTS reagents (Beck et al., 1999; Sobolevsky et al., 2002b). The accessibility of the deep positions in AMPAR channel to MTSEA is not caused by this reagent crossing the membrane in its uncharged form (Karlin and Akabas, 1998) (and hence reacting from the intracellular side of the membrane) because some of these positions (I-15, G-12, and $\mathrm{W}-10$ ) were accessible to MTSET, a permanently charged MTS reagent (MTSET + Glu), and the rate of reaction with MTSEA was strongly voltage dependent (see Fig. 4). Overall, the accessibility pattern in MTSET was more limited than that in MTSEA, presumably reflecting stronger steric constraints on accessibility to the larger-sized MTSET. The possibility of silent reactions for some of these differences cannot be excluded.

Steady-state accessibility of substituted cysteines is strongly state dependent (cf. MTSEA + Glu and MTSEA - Glu). The most notable feature is that no positions accessible to MTSEA below L-5 in the presence of glutamate are accessible in the absence of glutamate. Our interpretation of this difference is that the activation gate of the channel, presumably located between F-8 and L-5, allows access to the deeper positions when the channel is open and prevents their accessibility when the channel is closed (see below). Additional positions (I-2C, S-1C, N+4C, A+6C, V+11C, and $\mathrm{E}+12 \mathrm{C})$ also show state-dependent accessibility, with two of them $(\mathrm{V}+11 \mathrm{C}$ and $\mathrm{E}+12 \mathrm{C})$ accessible in the absence but not in the presence of glutamate. This strong state dependence suggests that the entire M3 segment and the region $\mathrm{C}$ terminal to it are involved in gating-related movements.

One common feature of the patterns shown in Figure $2 C$ is the lengthy region of accessible positions from $\mathrm{T}+2$ to $\mathrm{F}+8$. This region is a part of SYTANLAAF, which contains the "Lurcher" position (A+7) (Zuo et al., 1997) and is involved in coupling ligand binding to GluR channel opening (Kohda et al., 2000;
Jones et al., 2002). Supporting the idea of the importance of SYTANLAAF to channel gating, three positions in this motif in GluR-A $(\mathrm{A}+3, \mathrm{~N}+4$, and $\mathrm{L}+5)$ showed significant changes in the leak current amplitude before and after MTS exposure (Fig. 2D). The strongest effect was observed for $\mathrm{A}+3 \mathrm{C}$ where the large, compared with wild type, leak current amplitude was reduced more than twice after MTSEA application $(\Delta$ leak $=56 \pm 6 \% ; n=$ 3). Similar but reversible inhibition of the $A+3 \mathrm{C}$ leak current was produced by $2 \mu \mathrm{M}$ philanthotoxin $343(\Delta$ leak $=54 \pm 6 \% ; n=5)$, an AMPAR open channel blocker (Bahring and Mayer, 1998), supporting the idea that this leak current in oocytes injected with $\mathrm{A}+3 \mathrm{C}$ is mediated by AMPAR channels. Hence, $\mathrm{A}+3 \mathrm{C}$ substitution disrupts the closed state, an effect that we do not explore further here. 
Modification rate of cysteine-substituted AMPAR channels

Modification rates of substituted cysteines depend on a number of factors (Karlin and Akabas, 1998), including (among others) the local and global steric constraints such as the size of the waterfilled pathway leading up to the substituted cysteine; for charged reagents, the electrostatic potential along the pathway and at the residue; and the acid dissociation of the cysteine thiol group. Figure 3, $A$ and $B$, illustrates our protocols to measure modification rates in the presence or absence of glutamate. MTSEA (Fig. $3 A$, thick line, $1 \mathrm{~min}$ ) was applied five times either in the presence of glutamate (thin lines) or in the absence of glutamate but in the presence of the competitive AMPAR antagonist CNQX $(10 \mu \mathrm{M})$ (Fig. 3B, open boxes) at a holding potential of $-60 \mathrm{mV}$. Glutamate-activated current amplitudes, plotted as a function of the cumulative time of MTSEA exposure, were fit with a single exponential. The time constant of these fits defined the apparent second-order rate constant for chemical modification in the presence, $k$, and absence, $k_{\mathrm{CNQX}}$, of glutamate. For I-2C and S-1C, we show only the $k$ and $k_{\mathrm{CNQX}}$ values for the slow inhibitory component (Figs. 3, 4).

Figure $3 C$ summarizes the values of $k$ (solid circles) and $k_{\mathrm{CNQX}}$ (open circles) for selected exposed positions. These rates varied widely, but this variability is similar to that observed for NMDAR channels (Sobolevsky et al., 2002a,b) and probably reflects multiple factors that can affect $k$ and $k_{\mathrm{CNOX}}$ (see above). Notable is the difference between $k$ and $k_{\mathrm{CNQX}}$. With a single exception $(\mathrm{L}+5)$, modification of substituted cysteine in the presence of glutamate was always faster than in the absence of glutamate $\left(k>k_{\mathrm{CNOX}}\right)$. For certain positions, the state-dependent changes in modification rate could be caused by local changes in orientation of substituted cysteines, but in general they suggest that at least below $\mathrm{L}+5$ the whole AMPAR channel pore becomes less accessible with channel closure. In analogy to NMDAR (Sobolevsky et al., 2002a), the pore of AMPAR channel may become narrower in the closed than in the open state.

\section{Positioning of M3 relative to the tip of the M2 loop on the basis of voltage dependence of modification rates}

One approach to compare the relative positioning of exposed residues is to measure the voltage dependence of modification rates. To do so, we performed experiments as in Figure $3 A$ at different membrane potentials, $V_{\mathrm{h}}$. Figure $4 A$ shows two examples of rate constants, expressed in a logarithmic form $(-(R T)$ $\left.F)^{\star} \operatorname{Ln} k\right)$, as a function of $V_{\mathrm{h}}$. For I-15, the rate constants were strongly voltage dependent, getting faster at more negative potentials. In contrast, for $\mathrm{L}+5$, their voltage dependence was much weaker. The slope of the fitted line to plots such as those illustrated in Figure $4 A$ gives an estimation of $z \delta$, the fraction of the transmembrane electric field MTSEA passes to reach the exposed cysteine $(\delta)$ multiplied by the reagent charge $(z)$ (see Materials and Methods). Figure $4 B$ summarizes the $z \delta$ values for selected positions. The voltage dependence was the strongest for the presumed deepest position $\mathrm{I}-15(z \delta=1.08 \pm 0.06)$ and decreased monotonically as one moved more externally with the rates for $\mathrm{F}+8$ essentially voltage independent $(z \delta=0.02 \pm 0.03)$. The voltage dependence of the modification rate for the $\mathrm{Q} / \mathrm{R} / \mathrm{N}$ site in the M2 loop, Q582C $(z \delta=0.70 \pm 0.01)$, was comparable with that for $\mathrm{L}-5 \mathrm{C}(z \delta=0.73 \pm 0.02)$.

Clearly, the $z \delta$ values do not necessarily correspond to any physical distance. Nevertheless, taking into account the consistency of the overall voltage dependence for M3 - it drops uniformly from presumed deep to external positions-, we assume that the $z \delta$ values give an approximate index of the relative loca-
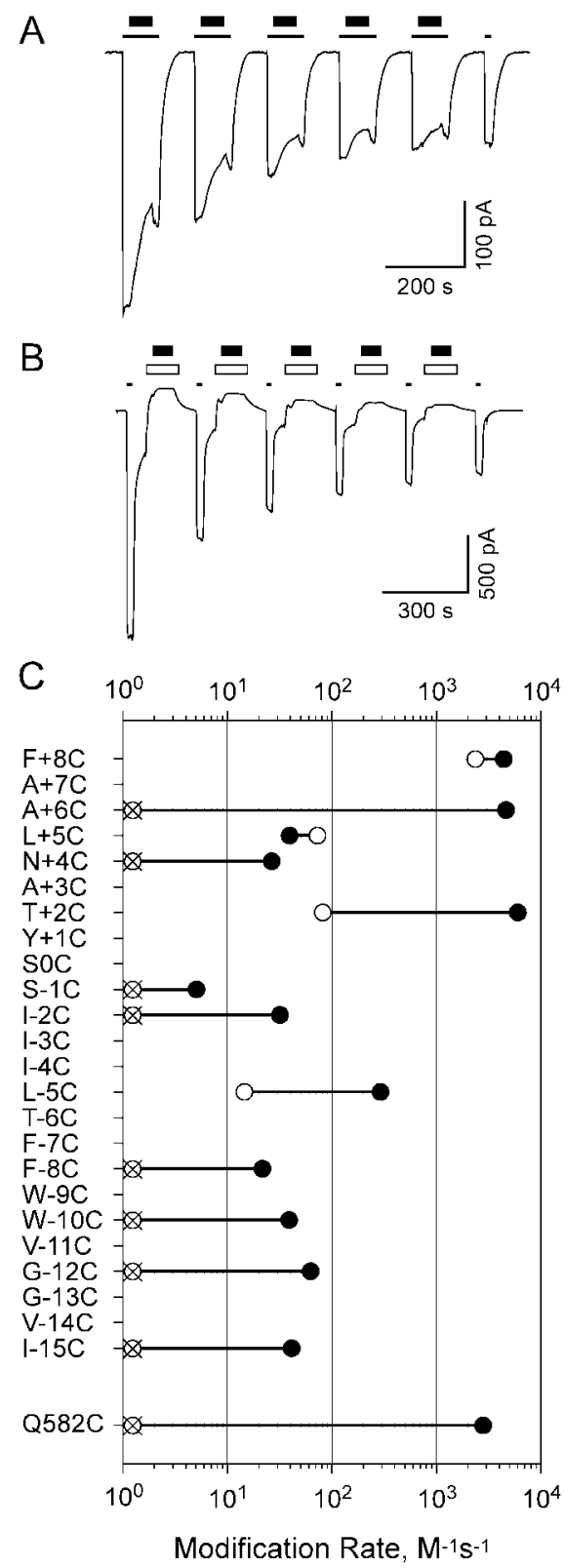

Figure 3. Modification rates of substituted cysteines in GluR-A channels. $A, B$, Pulsive protocols to assay modification rates of exposed cysteines in the presence $(A)$ or absence $(B)$ of glutamate. The examples show $\mathrm{T}+2 \mathrm{C}$ channels. $V_{\mathrm{h}}$ was $-60 \mathrm{mV}$. $A$, The MTSEA application (2 $\mu \mathrm{m}$, thick line, $1 \mathrm{~min}$ ) was started $15 \mathrm{sec}$ after the beginning and finished $15 \mathrm{sec}$ before the end of the glutamate (thin line) application. The cell was washed for 1.5 min between glutamate applications. Current amplitudes, defining the time course of cysteine modification, were measured during the first $15 \mathrm{sec}$ of each glutamate exposure. B, One minute after a 15 sec test glutamate application (thin line), CNQX (10 $\mu \mathrm{m}$, open box) was applied for $1.5 \mathrm{~min}$. The MTSEA application ( $200 \mu \mathrm{m}$, thick line, $1 \mathrm{~min}$ ) was started $15 \mathrm{sec}$ after the beginning and finished $15 \mathrm{sec}$ before the end of the CNQX exposure. After CNQX, the cell was washed for $1.25 \mathrm{~min}$ before the next test glutamate application. $C$, Apparent second-order rate constants for modification of substituted cysteines by MTSEA. Glutamate-activated current amplitudes $(A, B)$ were fitted with a single exponential. The time constants of these fits define the second-order rate constant for chemical modification of substituted cysteines. These rate constants measured in the presence $(k)$ or absence $\left(k_{\mathrm{CNOX}}\right)$ of glutamate are shown as solid and open circles, respectively. Crossed circles represent the $k_{\mathrm{CNQX}}$ values $<1 \mathrm{~m}^{-1} \mathrm{sec}^{-1}$. SEMs are smaller than the symbol size $(n>4)$.

tion of exposed positions in the AMPAR channel. On the basis of this assumption, the tip of the M2 loop (Q582) is located at the same approximate level as $\mathrm{L}-5$ in the M3 segment.

Supporting the above conclusion, the values of $k_{\mathrm{CNQX}}$ for 

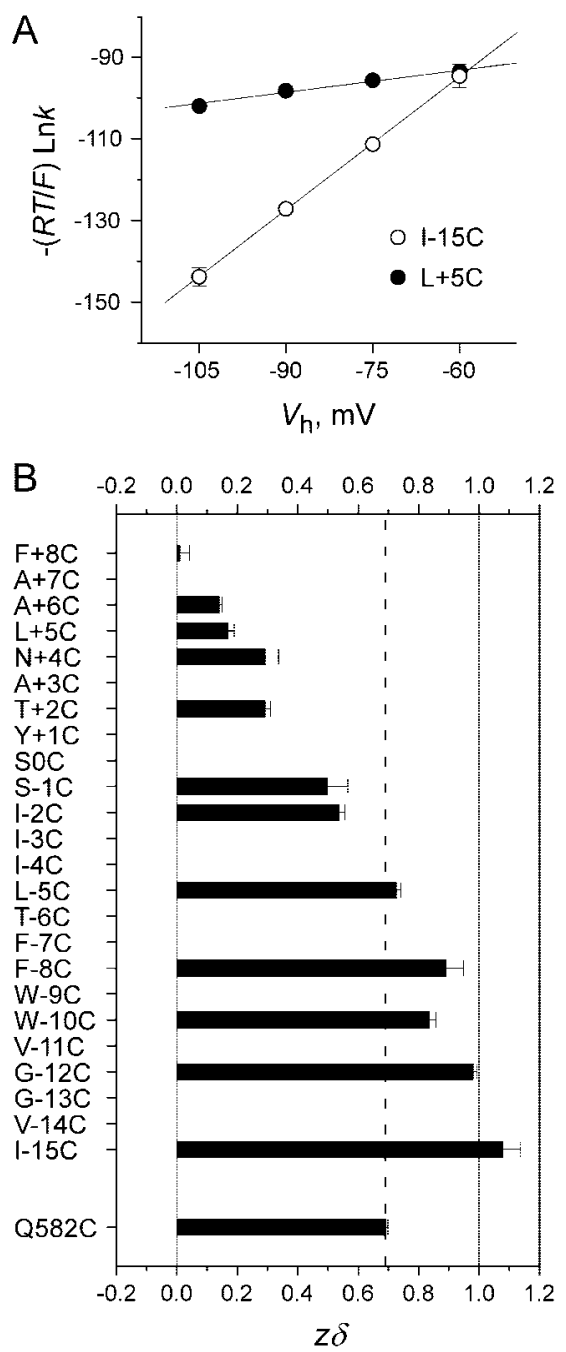

Figure 4. Voltage dependence of modification rate. $A$, Apparent second-order rate constant for MTSEA modification, expressed in a logarithmic form $\left(-(R T / F)^{*} \operatorname{Ln} k\right)$, as a function of the holding membrane potential, $V_{\mathrm{h}}$. The examples show $\mathrm{I}-15 \mathrm{C}(\mathrm{O})$ and $\mathrm{L}+5 \mathrm{C}(\mathbf{O})$. The $k$ values were estimated using the protocol illustrated in Figure $3 A$. Some error bars are smaller than the symbol size. The straight lines through the points are fits with Equation 2 (see Materials and Methods). The slopes of the fits give $z \delta: 1.08 \pm 0.06$ for $\mathrm{I}-15 \mathrm{C}$ and $0.18 \pm 0.02$ for $\mathrm{L}+5 \mathrm{C} . \mathrm{B}$, Mean $z \delta$ estimated using the method described in $A$. A minimum of four cells were recorded at each potential. The dashed line indicates the mean $z \delta$ value for $0582 C(0.70 \pm 0.01)$.

Q582C as well as for all positions located deeper than L-5 in M3 are $<1 \mathrm{M}^{-1} \mathrm{sec}^{-1}$ (Fig. 3C), suggesting that Q582 is located deeper than $\mathrm{L}-5$. On the other hand, the $k$ values for M3 positions located deeper than $\mathrm{L}-5 \mathrm{C}$ are always $<10^{2} \mathrm{M}^{-1} \mathrm{sec}^{-1}$, whereas that for Q582C is $>10^{3} \mathrm{M}^{-1} \mathrm{sec}^{-1}$, placing the Q/R site external to positions located deeper than $\mathrm{L}-5$. Taken together, both observations support the conclusion drawn from the voltage dependence experiments that Q582 is located close to position L-5 in the M3 segment.

\section{The M3 segment is $\alpha$-helical}

With the assumptions of SCAM in mind (see beginning of Results), accessibility of substituted cysteines to MTS reagents (Figs. 2,3 ) can address secondary structure. Figure $5 A$ shows a binary representation of the accessibility of substituted cysteines in M3 on an ideal $\alpha$-helix (helical wheel). We include in this analysis only positions in the M3 segment (positions less than +9 ) because those located C-terminal to M3 may be located outside the transmembrane part of AMPAR. Solid and open symbols in Figure $5 A$ indicate accessible and nonaccessible positions, respectively. In the presence of glutamate $(+\mathrm{Glu})$, the deep part of M3 (Fig. 5A, positions $\mathrm{I}-15$ to $\mathrm{T}+2$, top left wheel) shows a clear sidedness of accessibility consistent with this region forming an $\alpha$-helix. Indeed, with one exception (W-10), all accessible positions are located on the same side of the helical wheel. The accessibility of W-10C could reflect that it is located in a water-filled crevice or that the introduced cysteine disrupts pore structure. In the absence of glutamate ( - Glu; bottom left wheel), all positions below $\mathrm{L}-5$ are not accessible, presumably reflecting that they are located below the activation gate.

The more external part of M3 (positions I-2 to F+8) also demonstrates sidedness of accessibility, in both the presence (top right wheel) and absence (bottom right wheel) of glutamate. Compared with the deep part of M3, however, the line separating "accessible" and "nonaccessible" sides of the helical wheel has a different orientation. This change in orientation could be attributable to a tilt of M3 relative to the central axis of the pore (Fig. 7) or to a complex packing of M1 and M4 with respect to M3, or both. Another notable feature is that the accessible side of the helical wheel in the presence of glutamate $(+$ Glu) is much larger than that in the absence of glutamate $(-\mathrm{Glu})$. This reduction of the surface area, specifically on both edges of the accessible side, is consistent with the idea that there is a narrowing of the entire extent of the extracellular vestibule with channel closure (Sobolevsky et al., 2002a). Finally, mutations that disrupt the closed state, $\mathrm{A}+3$ (present study) and $\mathrm{A}+7$ (Lurcher position, Kohda et al., 2000) are located on the nonaccessible side of the helix.

In summary, the good correspondence between the experimental data and the ideal model strongly suggests that the M3 segment in AMPAR channel forms a pore-lining $\alpha$-helix like the homologous domain $\mathrm{M} 2$ in $\mathrm{K}^{+}$channels.

\section{Vertical alignment of GluR subunits}

To compare the relative positioning of GluR subunits (NR1, NR2C, and GluR-A), we aligned their M3 segments along the central axis of the channel pore (Fig. $5 B$ ) by placing the tip of the M2 loop (the Q/R/N site) at the same approximate level (dashed line). The vertical positioning of the M3 segments relative to this level is on the basis of observations that the $Q / R / N$ site in NR1 (N598) is close to V-2 (Beck et al., 1999; Sobolevsky et al., 2002a); in NR2C (N593) it is close to V-5 (Sobolevsky et al., 2002a), and in GluR-A (Q582) it is close to L-5 (see above).

The M3 segments in NMDAR NR1 and NR2C subunits are staggered with positions in NR2C located about four amino acid residues more externally than homologous ones in NR1 (Sobolevsky et al., 2002b). In terms of this relative positioning, M3 in GluR-A, where the -5 position is external to the tip of the M2 loop, is more NR2C-like. Also notable is that the accessibility of positions located deeper than $\mathrm{N}+4$ is identical for NR2C in the presence of glutamate (NR2C, + Glu) and for GluR-A in the absence of glutamate (AMPA, -Glu). Nevertheless, in detail, GluR-A M3 shows a distinct pattern of accessibility relative to NMDAR subunits (e.g., position -1 is accessible in GluR-A but not in either NMDAR subunits). In addition, AMPAR subunits may be staggered relative to each other, but this staggering is different from NMDARs because the pattern of accessibility is not the weighted sum of those seen in NMDAR subunits.

The greatest difference between GluR subunits is the accessibility of the deep regions in the presence of glutamate. For NMDAR subunits, no residues located below the tip of the M2 loop ( $\mathrm{N}$ site) are accessible in the presence of glutamate. In con- 
A
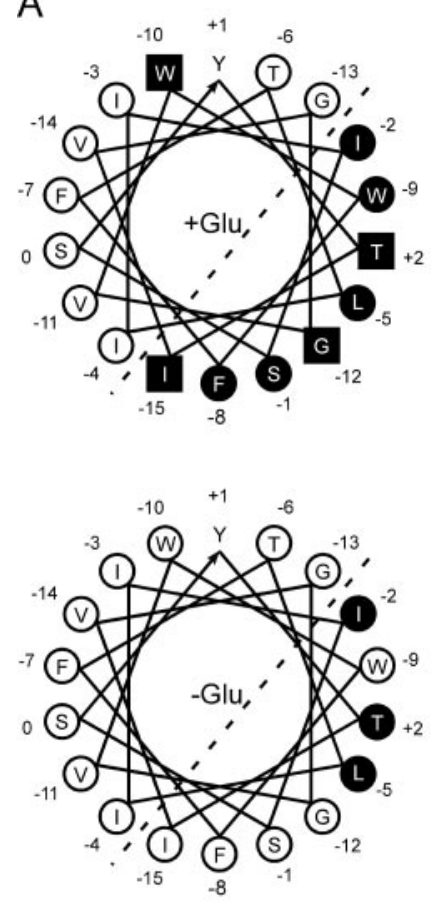
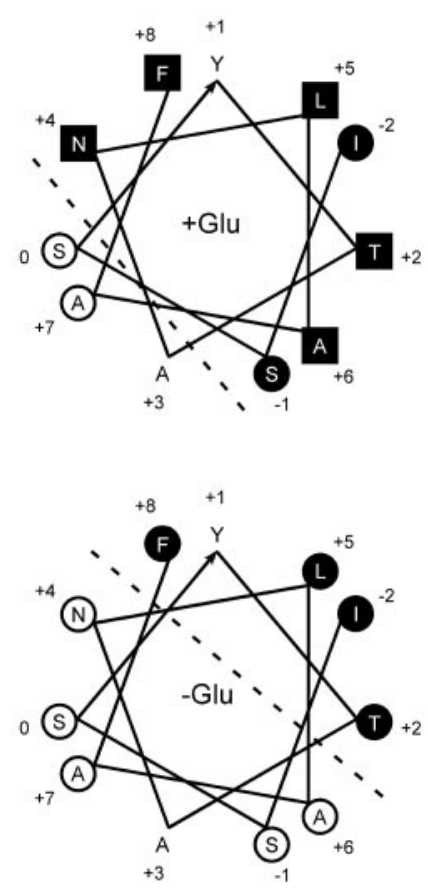

B

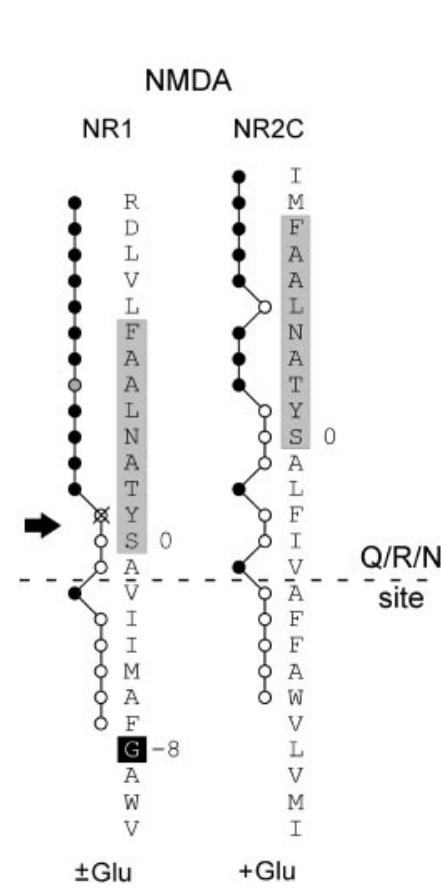

AMPA

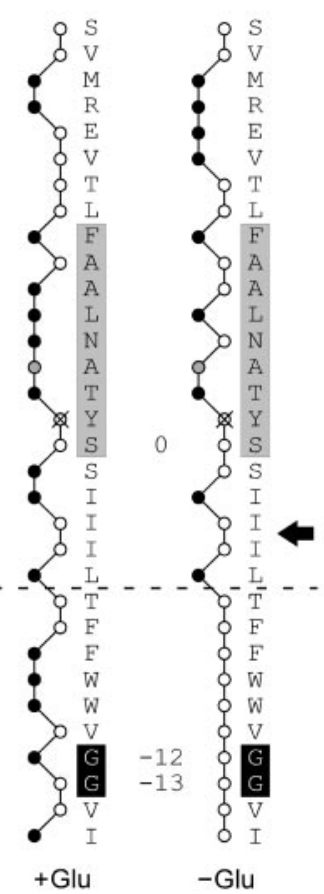

Figure 5. $\alpha$-Helical structure and vertical positioning of the M3 segment. A, Accessibility of substituted cysteines in M3 suggests an $\alpha$-helical secondary structure. Helical wheel analysis of accessibility of cysteines substituted at positions -15 to +2 (left wheels) and -2 to +8 (right wheels) in GluR-A subunit in the presence (top wheels) or absence (bottom wheels) of glutamate. We do not include regions (-terminal to $\mathrm{M} 3$ (positions more than +8 ) in this analysis because they may be located outside the transmembrane region having all sides water accessible. Open circles indicate nonaccessible positions. Black circles indicate positions accessible to MTSEA. Black squares indicate positions accessible to both MTSEA and MTSET in the presence of glutamate. Dashed line separates accessible and nonaccessible sides of the helical wheel. $Y+1 C$ does not generate a detectable glutamate-activated current. $A+3 C$ forms channels with large leak currents, limiting the interpretation of cysteine modification. B, Vertical alignment of GluR subunits. Binary representation of the accessibility of substituted cysteines to MTS reagents in the NR1, NR2C, and GluR-A M3 segments in the presence (+Glu) or absence (-Glu) of glutamate. A position is considered accessible (filled circle) if at least one MTS reagent (MTSEA, MTSET, or 3-(triethylammonium)propyl methane thiosulfonate) produced a significant alteration in glutamate-activated current. Other positions are represented as open circles. The NR1 results are from Beck et al. (1999) and Sobolevsky et al. (2002a), whereas those for NR2C are from Sobolevsky et al. (2002b). Y+1C mutant in NR1 and GluR-A does not produce functional channels (crossed circle). GluR-A(A + 3C) and NR1(A + 6C)NR2C channels exhibit large leak current and a strong and irreversible inward current after MTS exposure, respectively (gray circles). The approximate location of the Q/R/N site or the tip of the M2 loop relative to $\mathrm{M} 3$ is indicated by the dashed line. In a pure structural homology to an inverted $\mathrm{K}^{+}$channel, the glycine "gating hinge" would be located right above the tip of the reentrant loop (black arrows).

trast, numerous positions located deeper than the $\mathrm{Q} / \mathrm{R}$ site in GluR-A (I-15, G-12, W-10, W-9, and F-8) are accessible. The basis for the accessibility of these deep positions is unknown but it may be attributable to permeation of MTS reagents through the pore of open AMPAR channel or a less dense packing of poreforming domains. Alternatively, cysteine substitutions of the deep M3 positions might disrupt the structure of the pore making an endogenous cysteine (e.g., C585 in M2) accessible to externally applied MTS reagents. We think this alternative is unlikely because of the strong voltage dependence of reaction rates (Fig. $4 B$ ) and strong sidedness of accessibility (Fig. 5A).

\section{No glycine gating hinge in the GluR-A M3 segment}

The gating hinge in $\mathrm{K}^{+}$channels is represented by a highly conserved glycine, the only amino acid residue [along with proline (Javadpour et al., 1999)] that allows for flexibility in an $\alpha$-helix. The key feature of this glycine is its location just below the tip of the P loop (Fig. 1) (see Introduction). In a strict homology to $\mathrm{K}^{+}$ channels and taking into account the inverted membrane topology, a gating hinge glycine in GluR would be located one to three positions external to the tip of the M2 loop (the Q/R/N site). In GluR-A, this point corresponds to one of three isoleucines, I-4, I-5, or I-6 (Fig. 5B, black arrows). Indeed, neither GluR-A nor NR1 or NR2C have a glycine residue located right above or even close to the tip of the M2 loop. The closest glycines in the GluR-A M3 segment are located six and seven positions (9 and $10.5 \AA$ ) deeper than the tip of the M2 loop (Fig. 5B, black boxes). To address whether G-12 and G-13 form a gating hinge in GluR-A channels, we mutated these glycines to alanines, which would remove flexibility in an $\alpha$-helix.

In $\mathrm{K}^{+}$channels, alanine substitutions of the gating hinge glycine yield nonfunctional channels (Yifrach and MacKinnon, 2002; Ding and Horn, 2003). In contrast, both leak and glutamate-activated current amplitudes in G-12A/G-13A GluR-A channels were similar to those for wild-type channels. In addition, all stationary and kinetic parameters for wild-type and G-12A/G-13A channels were indistinguishable, including the time constants for the entrance $\left(\tau_{\mathrm{des}}\right)$ and recovery $\left(\tau_{\text {rec }}\right)$ from desensitization, the ratio of the steady-state to peak current amplitudes (SS/peak ratio), and the deactivation time constant $\left(\tau_{\text {deact }}\right)$ (Fig. 6, Table 1). Concentration-response curves, in this instance for channels in a nondesensitizing L479Y background and hence measured for the plateau current, were similarly indistinguishable (Fig. 6B). Therefore, G-12 and G-13 glycines do not make any significant contribution to channel gating. Because these two glycines are the only ones in the GluR-A subunit M3 segment, we conclude that this $\alpha$-helical domain does not contain a glycine gating hinge. 

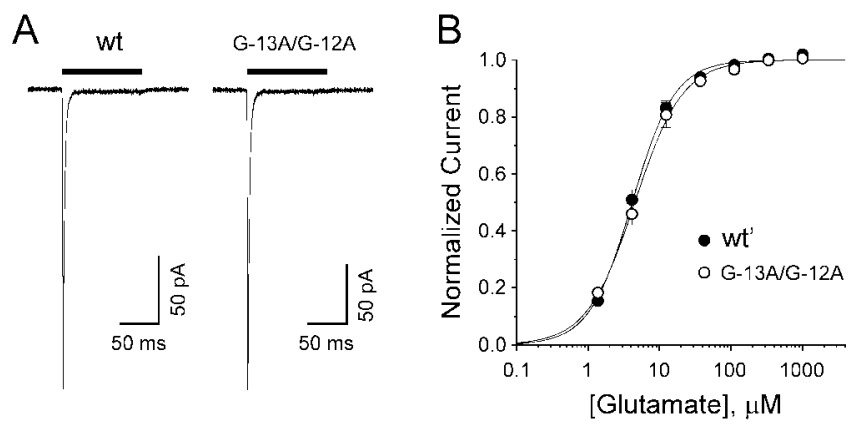

Figure 6. Gating kinetics and concentration dependence of glutamate-activated currents for wild-type and $\mathrm{G}-13 \mathrm{~A} / \mathrm{G}-12 \mathrm{~A}$ channels. $A$, Currents recorded from outside-out patches excised from HEK 293 cells expressing wild-type or $G-12 A / G-13 A$ GluR-A channels (the subunits did not contain the nondesensitizing L479Y mutation). Currents were elicited by a $100 \mathrm{msec}$ pulse of glutamate ( $1 \mathrm{~mm}$, thick bar) using fast agonist exchange. Holding potential was -60 $\mathrm{mV}$. $B$, Concentration-response curves for glutamate-activated currents recorded from oocytes expressing wild-type (wt') or G-13A/G-12A channels in the nondesensitizing background (L479Y). Solid lines are fits of the data with the Hill equation, yielding $\mathrm{EC}_{50}$ and $n_{\text {Hill }}$ values of $4.1 \pm 0.2 \mu \mathrm{m}$ and $1.46 \pm 0.07$ for $\mathrm{wt}^{\prime}(n=5)$ and $4.5 \pm 0.2 \mu \mathrm{m}$ and $1.30 \pm 0.05$ for $\mathrm{G}-13 \mathrm{~A} / \mathrm{G}-12 \mathrm{~A}(n=6)$

Table 1. Basic gating characteristics of wild-type and mutant (G-13A/G-12A) GluR-A channels

\begin{tabular}{lcccc}
\hline & $\tau_{\text {des }}$ msec & $\tau_{\text {rec }}$ msec & SS/peak ratio & $\tau_{\text {deact }}$ msec \\
\hline Wild type & $3.1 \pm 0.3$ & $162 \pm 13$ & $0.016 \pm 0.003$ & $0.83 \pm 0.07$ \\
G-13A/G-12A & $3.2 \pm 0.2$ & $159 \pm 16$ & $0.014 \pm 0.002$ & $0.79 \pm 0.09$
\end{tabular}

Time constants for the entrance ( $\left.\tau_{\text {des }}\right)$ and recovery $\left(\tau_{\text {rec }}\right)$ from desensitization, plateau/peak ratios (SS/peak ratios) and deactivation time constants ( $\tau_{\text {deact }}$ ) were measured in outside-out patches excised from HEK 293 cells ( $n=$ $4-10$; see Materials and Methods for details).

\section{Discussion}

In the present study, we took advantage of substituted cysteines and fast agonist application to study the structure of poreforming domains in AMPARs, specifically those composed of GluR-A subunits. We focused mainly on the spatial positioning and functional significance of glycines in the M3 segment because of their prominence to the gating mechanism in $\mathrm{K}^{+}$channels. We find that AMPARs show general structural similarities to $\mathrm{K}^{+}$ channels, with the M3 segment, like the homologous domain in $\mathrm{K}^{+}$channels, $\alpha$-helical in structure, lining the channel pore and involved in gating; however, the M3 segment does not have a glycine residue located right above or even close to the tip of the M2 loop (Fig. 5B), and glycines in M3 do not contribute to channel gating (Fig. 6). This absence of a glycine gating hinge may represent the structural basis for the distinct features of gating in GluRs compared with $\mathrm{K}^{+}$channels.

\section{The M3 segment in GluR subunits does not contain a glycine gating hinge}

The M3 segments in AMPAR and KAR subunits are highly conserved, suggesting that like GluR-A, no non-NMDAR subunit has a glycine gating hinge in M3. Neither NR1 nor NR2C subunits have a glycine located externally to the tip of the M2 loop (Fig. $5 B$ ), arguing against a functional similarity to $\mathrm{K}^{+}$channels. In fact, the entire M3 of any NR2 or NR3 subunits does not contain a single glycine residue. On the other hand, NR1 does have a glycine at about the same location as in GluR-A (Fig. 5B). The secondary structure of M3 in NMDAR subunits is unknown, but presumably is $\alpha$-helical. Therefore, taking into account the high sequence similarity of the M3 segments and assuming similarity in general design of structure and gating of different GluR subtypes, it seems unlikely that any GluR channel subunit has a glycine gating hinge in M3.

What is the significance of the glycines in the N-terminal part of M3, which are to some extent conserved in GluR subunits (Fig. $1 B)$ ? Although unknown, one possibility is that these glycines represent important contact points between helices. Indeed, glycines as well as other small side-chain amino acids (including alanines) are prevalent at helix-helix interfaces of membrane proteins (Eilers et al., 2002). Given the positioning of these glycines below the tip of the M2 loop in GluR subunits, such a function seems likely (and the glycine to alanine substitution would be expected to introduce minor changes in this function). Nevertheless, more extensive mutagenesis will be required to fully address the structural significance of glycines in M3 as well as other transmembrane domains.

\section{Location of the activation gate}

In the M3 segment of GluR-A, many of the exposed positions located external to the tip of the M2 loop $(\mathrm{L}-5, \mathrm{I}-2, \mathrm{~T}+2, \mathrm{~A}+3$, $\mathrm{L}+5, \mathrm{~F}+8, \mathrm{R}+13, \mathrm{M}+14$ ) show a state-dependent accessibility (Fig. $3 B$ ) but remain accessible even in the absence of glutamate (Figs. 2C,3B). This pattern of accessibility is comparable with that found in the NMDAR NR1 subunit (Beck et al., 1999; Sobolevsky et al., 2002a) and in analogy suggests that the activation gate, the structure that occludes the flux of permeant ions in the closed state, is not located in the extracellular vestibule but rather is positioned at the narrow constriction of the channel (the tip of the M2 loop) or deeper. Further supporting this idea for GluR-A channels is that a consecutive string of positions located below $\mathrm{L}-5$ are not accessible in the absence of glutamate even for steadystate reactions (Fig. 2C).

In voltage-gated and bacterial $\mathrm{K}^{+}$channels, the activation gate is formed by the bundle crossing of the inner helices with the narrowest part around A111 in KcsA (Liu et al., 1997; del Camino and Yellen, 2001; Jiang et al., 2002b). The homologous location in GluR would be the extracellular end of M3 (position F+8 in NR2C and GluR-A and position D+12 in NR1), at least 13 residues (19.5 $\AA$ ) external to the tip of the M2 loop. Our results, therefore, support the idea that the location of the activation gate in GluR channels is different from voltage-gated and bacterial $\mathrm{K}^{+}$ channels but similar to CNG (Sun et al., 1996; Flynn and Zagotta, 2001) and small conductance $\mathrm{Ca}^{2+}$-activated $\mathrm{K}^{+}$channels (Bruening-Wright et al., 2002), where it is associated with the reentrant $\mathrm{P}$ loop. It is also interesting to note that both AMPAR (Rosenmund et al., 1998; Smith and Howe, 2000) and CNG (Ruiz and Karpen, 1997) channels show concentration-dependent subconductance levels. Nevertheless, mechanisms underlying movement of the reentrant pore loops in channels with a glycine gating hinge and those without may or may not be comparable.

\section{Structural similarity but different gating mechanisms in GluR and $\mathrm{K}^{+}$channels}

$\mathrm{K}^{+}$and GluR channels share a common structural design, having a similar, although inverted, membrane topology (Wo and Oswald, 1995; Wood et al., 1995) (Fig. 1 A), an idea supported by the discovery of the prokaryotic glutamate receptor GluR0 that forms a possible evolutionary link between GluR and $\mathrm{K}^{+}$channels (Chen et al., 1999). The strongest evidence for this structural homology is for the reentrant M2 loop in GluRs that includes an $\alpha$-helix followed by an extended region (Kuner et al., 1996, 2001; Panchenko et al., 2001) like the corresponding domain (P loop) in $\mathrm{K}^{+}$channels (Doyle et al., 1998; Jiang et al., 2002a). The homologous domains M2 in bacterial $\mathrm{K}^{+}$channels (S6 in voltage- 


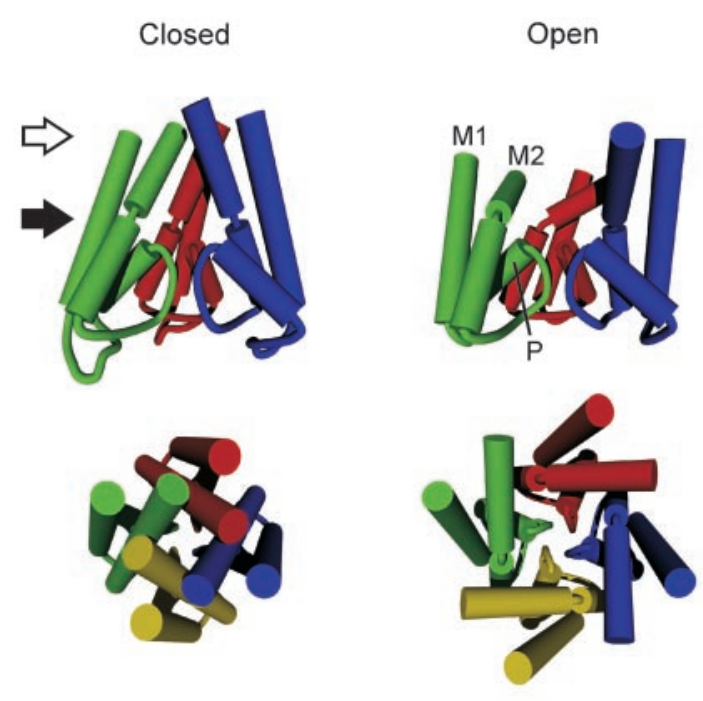

$\mathrm{K}^{+}$channel

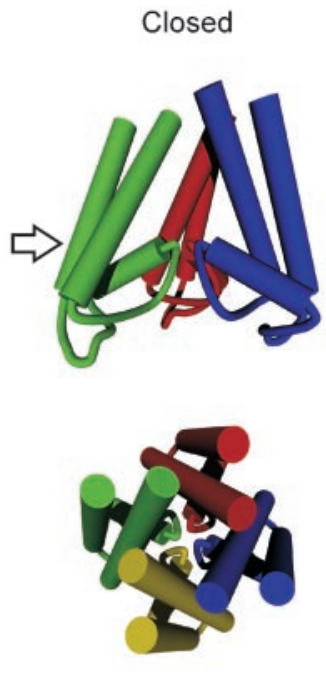

Open

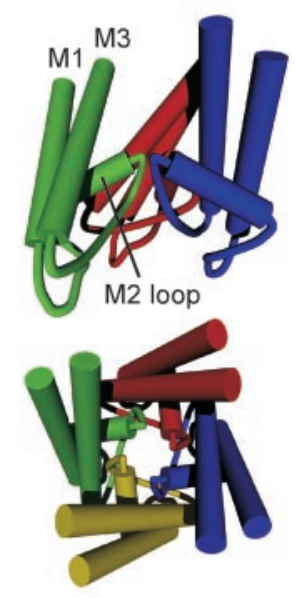

GluR channel

Figure 7. Different gating mechanisms in $\mathrm{K}^{+}$and GluR channels. Models of $\mathrm{K}^{+}$(left) and $\mathrm{GluR}$ (right) channels in the closed or open conformations. Each column contains two diagrams that illustrate the channel viewed from the side (top) or from either the intracellular ( $\mathrm{K}^{+}$channel) or extracellular (GluR channel) side of the membrane (bottom). In side views, the front subunit is removed and the other three are oriented with the intracellular and extracellular surfaces on the top for $\mathrm{K}^{+}$and GluR channels, respectively. KcsA (Doyle et al., 1998) and MthK (Jiang et al., 2002b) structures represent the closed and open conformations of $\mathrm{K}^{+}$channel, respectively. Only the M1, M2, and M3 segments are shown for GluR channel. White and black arrows indicate the approximate gate levels and the gating hinge in $\mathrm{K}^{+}$channel, respectively.

gated $\mathrm{K}^{+}$channels) (Jiang et al., 2003a,b) and M3 in GluR channels line the intracellular and extracellular vestibules, respectively, and are involved in channel gating. Our results also indicate that positioning of the tip of the M2 loop relative to M3 is comparable with that for homologous domains in $\mathrm{K}^{+}$channels. Nevertheless, the M3 helices in GluRs do not contain a gating hinge, the principle structural element defining $\mathrm{K}^{+}$channel gating. On the basis of these similarities and differences, we modified the model of $\mathrm{K}^{+}$channel gating (see Materials and Methods) to create a general structural model of channel gating in GluRs (Fig. 7).

The two left panels in Figure 7 are diagrams illustrating key features of $\mathrm{K}^{+}$channel gating (Jiang et al., 2002b). In this model, the crystal structures of KcsA (Doyle et al., 1998) and MthK (Jiang et al., 2002a) represent the closed and open conformations, respectively. In the closed state, the inner helices M2 are nearly straight. Their bundle crossing at the intracellular side of the membrane forms the activation gate that defines a large central cavity extending to the selectivity filter. In the open state, the M2 helices are bent at the glycine gating hinge and splayed open, forming a wide intracellular vestibule. Hence, the intracellular part of M2 moves extensively during gating. In contrast, the extracellular part, specifically that in contact with the P loop, remains essentially motionless, allowing the diameter of the selectivity filter, at least in voltage-gated and bacterial $\mathrm{K}^{+}$channels (Jiang et al., 2002b), to stay relatively stable and state independent.

Our model of GluR channel gating (Fig. 7, right panels) is similar to that for $\mathrm{K}^{+}$channels, including retaining a basic structural homology (see above). In addition, the general direction and character of movement of M1 and M3 during channel opening (they tilt relative to the central axis of the channel and splay out) remain similar to those for $\mathrm{M} 1$ and $\mathrm{M} 2$ in $\mathrm{K}^{+}$channels (Fig. 7 , left and right panels). The model differs from that for $\mathrm{K}^{+}$ channels, however, in that regardless of the activation state of the GluR channel, the extracellular vestibule is wide enough to ac- commodate MTS reagents (Fig. 2). An additional key difference is that the GluR M3 segment does not contain a gating hinge and accordingly is fairly rigid, causing it to tilt but not kink in the middle when the channel opens. Therefore, in contrast to $\mathrm{K}^{+}$ channels, the entire M3 including parts in contact with M2 move during gating. Because M2 and M3 are in physical contact, our model predicts that movement of M3 leads directly to movement of M2. Because M2 forms the narrowest part of the GluR channel pore, even subtle changes in its conformation cause occlusion/ opening of the channel pore during gating. We therefore hypothesize that it is the lack of a gating hinge in GluR channels that leads to the activation gate being located at the tip of the M2 loop or deeper.

In summary, we propose that the absence of a gating hinge in the GluR channel and its presence in the $\mathrm{K}^{+}$channel is the major reason for differences in their gating mechanisms. The model of GluR channel gating shown in Figure 7 is simplified and does not take into account detailed structural differences between GluR and $\mathrm{K}^{+}$channels as well as possible structural differences between GluR subtypes. Such differences include the presence of the M4 segment, possible two- rather than fourfold symmetry (dimer of dimers organization) (Armstrong and Gouaux, 2000; Robert et al., 2001), and the staggering (Sobolevsky et al., 2002b) and the order (Schorge and Colquhoun, 2003) of subunits in heteromeric complexes. The role of all of these factors as well as others needs to be characterized to fully define gating in GluR channels.

\section{References}

Armstrong N, Gouaux E (2000) Mechanisms for activation and antagonism of an AMPA-sensitive glutamate receptor: crystal structures of the GluR2 ligand binding core. Neuron 28:165-181.

Armstrong N, Sun Y, Chen GQ, Gouaux E (1998) Structure of a glutamatereceptor ligand-binding core in complex with kainate. Nature 395:913-917.

Bahring R, Mayer ML (1998) An analysis of philanthotoxin block for re- 
combinant rat GluR6(Q) glutamate receptor channels. J Physiol (Lond) 509:635-650.

Beck C, Wollmuth LP, Seeburg PH, Sakmann B, Kuner T (1999) NMDAR channel segments forming the extracellular vestibule inferred from the accessibility of substituted cysteines. Neuron 22:559-570.

Bruening-Wright A, Schumacher MA, Adelman JP, Maylie J (2002) Localization of the activation gate for small conductance $\mathrm{Ca}^{2+}$-activated $\mathrm{K}^{+}$ channels. J Neurosci 22:6499-6506.

Burnashev N, Villarroel A, Sakmann B (1996) Dimensions and ion selectivity of recombinant AMPA and kainate receptor channels and their dependence on Q/R site residues. J Physiol (Lond) 496:165-173.

Chen GQ, Cui C, Mayer ML, Gouaux E (1999) Functional characterization of a potassium-selective prokaryotic glutamate receptor. Nature 402:817-821.

del Camino D, Yellen G (2001) Tight steric closure at the intracellular activation gate of a voltage-gated $\mathrm{K}^{+}$channel. Neuron 32:649-656.

Ding S, Horn R (2003) Glycine scan of Shaker's gating hinge. Biophys J 84:73A.

Doyle DA, Morais Cabral J, Pfuetzner RA, Kuo A, Gulbis JM, Cohen SL, Chait BT, MacKinnon R (1998) The structure of the potassium channel: molecular basis of $\mathrm{K}^{+}$conduction and selectivity. Science 280:69-77.

Eilers M, Patel AB, Liu W, Smith SO (2002) Comparison of helix interactions in membrane and soluble alpha-bundle proteins. Biophys J 82:2720-2736.

Flynn GE, Zagotta WN (2001) Conformational changes in S6 coupled to the opening of cyclic nucleotide-gated channels. Neuron 30:689-698.

Javadpour MM, Eilers M, Groesbeek M, Smith SO (1999) Helix packing in polytopic membrane proteins: role of glycine in transmembrane helix association. Biophys J 77:1609-1618.

Jiang Y, Lee A, Chen J, Cadene M, Chait BT, MacKinnon R (2002a) Crystal structure and mechanism of a calcium-gated potassium channel. Nature 417:515-522.

Jiang Y, Lee A, Chen J, Cadene M, Chait BT, MacKinnon R (2002b) The open pore conformation of potassium channels. Nature 417:523-526.

Jiang Y, Lee A, Chen J, Ruta V, Cadene M, Chait BT, MacKinnon R (2003a) $\mathrm{X}$-ray structure of a voltage-dependent $\mathrm{K}^{+}$channel. Nature 423:33-41.

Jiang Y, Ruta V, Chen J, Lee A, MacKinnon R (2003b) The principle of gating charge movement in a voltage-dependent $\mathrm{K}^{+}$channel. Nature 423:42-48.

Jones KS, VanDongen HM, VanDongen AM (2002) The NMDA receptor M3 segment is a conserved transduction element coupling ligand binding to channel opening. J Neurosci 22:2044-2053.

Karlin A, Akabas MH (1998) Substituted-cysteine accessibility method. Methods Enzymol 293:123-145.

Kohda K, Wang Y, Yuzaki M (2000) Mutation of a glutamate receptor motif reveals its role in gating and delta2 receptor channel properties. Nat Neurosci 3:315-322.

Kuner T, Wollmuth LP, Karlin A, Seeburg PH, Sakmann B (1996) Structure of the NMDA receptor channel M2 segment inferred from the accessibility of substituted cysteines. Neuron 17:343-352.

Kuner T, Beck C, Sakmann B, Seeburg PH (2001) Channel-lining residues of the AMPA receptor M2 segment: structural environment of the $Q / R$ site and identification of the selectivity filter. J Neurosci 21:4162-4172.

Kuner T, Seeburg PH, Guy HR (2003) A common architecture for $\mathrm{K}^{+}$channels and ionotropic glutamate receptors? Trends Neurosci 26:27-32.

Liu Y, Holmgren M, Jurman ME, Yellen G (1997) Gated access to the pore of a voltage-dependent $\mathrm{K}^{+}$channel. Neuron 19:175-184.

Mayer ML, Olson R, Gouaux E (2001) Mechanisms for ligand binding to GluR0 ion channels: crystal structures of the glutamate and serine complexes and a closed apo state. J Mol Biol 311:815-836.

Panchenko VA, Glasser CR, Mayer ML (2001) Structural similarities between glutamate receptor channels and $\mathrm{K}^{+}$channels examined by scanning mutagenesis. J Gen Physiol 117:345-360.

Robert A, Irizarry SN, Hughes TE, Howe JR (2001) Subunit interactions and AMPA receptor desensitization. J Neurosci 21:5574-5586.

Rosenmund C, Stern-Bach Y, Stevens CF (1998) The tetrameric structure of a glutamate receptor channel. Science 280:1596-1599.

Ruiz ML, Karpen JW (1997) Single cyclic nucleotide-gated channels locked in different ligand-bound states. Nature 389:389-392.

Schorge S, Colquhoun D (2003) Studies of NMDA receptor function and stoichiometry with truncated and tandem subunits. J Neurosci 23:1151-1158.

Smith TC, Howe JR (2000) Concentration-dependent substate behavior of native AMPA receptors. Nat Neurosci 3:992-997.

Sobolevsky AI, Beck C, Wollmuth LP (2002a) Molecular rearrangements of the extracellular vestibule in NMDAR channels during gating. Neuron 33:75-85.

Sobolevsky AI, Rooney L, Wollmuth LP (2002b) Staggering of subunits in NMDAR channels. Biophys J 83:3304-3314.

Stern-Bach Y, Russo S, Neuman M, Rosenmund C (1998) A point mutation in the glutamate binding site blocks desensitization of AMPA receptors. Neuron 21:907-918.

Sun Y, Olson R, Horning M, Armstrong N, Mayer M, Gouaux E (2002) Mechanism of glutamate receptor desensitization. Nature 417:245-253.

Sun ZP, Akabas MH, Goulding EH, Karlin A, Siegelbaum SA (1996) Exposure of residues in the cyclic nucleotide-gated channel pore: $\mathrm{P}$ region structure and function in gating. Neuron 16:141-149.

Wo ZG, Oswald RE (1995) Unraveling the modular design of glutamategated ion channels. Trends Neurosci 18:161-168.

Wollmuth LP, Kuner T, Seeburg PH, Sakmann B (1996) Differential contribution of the NR1- and NR2A-subunits to the selectivity filter of recombinant NMDA receptor channels. J Physiol (Lond) 491:779-797.

Wood MW, VanDongen HM, VanDongen AM (1995) Structural conservation of ion conduction pathways in $\mathrm{K}$ channels and glutamate receptors. Proc Natl Acad Sci USA 92:4882-4886.

Yifrach O, MacKinnon R (2002) Energetics of pore opening in a voltagegated $\mathrm{K}^{+}$channel. Cell 111:231-239.

Zhou Y, Morais-Cabral JH, Kaufman A, MacKinnon R (2001) Chemistry of ion coordination and hydration revealed by a $\mathrm{K}^{+}$channel-Fab complex at 2.0 A resolution. Nature 414:43-48.

Zuo J, De Jager PL, Takahashi KA, Jiang W, Linden DJ, Heintz N (1997) Neurodegeneration in Lurcher mice caused by mutation in delta2 glutamate receptor gene. Nature 388:769-773. 